\title{
Synthesis and Implementation (In STM8S) of Phased Circular Antenna Arrays Using Taguchi Method
}

\author{
N. Nemri, H. W. Badri, R. Ghayoula, H. Trabelsi, A. Gharsallah \\ Unit of Research in High Frequency Electronic Circuits and Systems, Tunis El Manar University, Tunisia
}

\begin{tabular}{l} 
Article Info \\
\hline Article history: \\
Received Oct 13, 2015 \\
Revised Jan 1, 2016 \\
Accepted Jan 16, 2016 \\
\hline Keyword: \\
Circular antenna array \\
Optimisation \\
Radiation \\
Smart antenna \\
Taguchi's Method
\end{tabular}

\begin{abstract}
This paper is aimed at assessing the effectiveness of the phase-only control strategy based on a customized Taguchi method when applied to Uniform Circular Arrays (UCA). The objective of this paper consists to contribute the main lobe optimization of the smart antenna using Taguchi's method. We used the cited method in order to determine phase's weights for each element of the circular antenna array in order to steer the principal lobe from $-65^{\circ}$ to $65^{\circ}$ covering all angular space. After that, we made an electronic platform using the microcontroller STM8S in order to implement an intelligent system. The architecture of this work had used a digital phase shifters, a demodulator AD8347, a modulator AD8349, an array antenna, cards STM8S-Discovery.
\end{abstract}

Copyright () 2016 Institute of Advanced Engineering and Science. All rights reserved.

Corresponding Author:

Nadhem Nemri,

Unit of Research in High Frequency Electronic Circuits and Systems,

Tunis El Manar University, Tunisia

Email: nadhem.nemri@gmail.com

\section{INTRODUCTION}

In the last few years, in some countries the number of subscribers to wireless services has boosted greatly. Actually, more than $60 \%$ of the population are enjoying it with no sign of saturation. Providing everyone, everywhere at anytime with mobile communication service at low cost with high quality and high data rates but with low emission of radiation and so forth has become à preoccupation. This leads to challenges for the system designer as these objectives are counteracting. Over the last decade, smart antenna systems are required in the communications industry due to their adaptive features. Smart antennas can be categorized into two main techniques; switched beam array and phased array antennas. In a switched beam array, the direction of the beam is chosen from a set of predetermined beams while in a phased array, the main beam is steered towards a specific direction. Circular antenna arrays have been developed and presented for a variety of applications: in mobile and commercial satellite communications systems, sonar and radar. It consists of a number of elements arranged on a circle [1] with uniform spacing and can be used for beam forming in the azimuth plane for example at the base stations of the mobile radio communications system [2]. They are very useful in high power transmission, reduced power consumption and enhanced spectral efficiency. To provide a very directive pattern, it is necessary that the fields from the array elements must add constructively in some desired direction and add destructively and cancel each other in the remaining space. This is important to reduce interference from side lobe of the antenna [3].

Among the different type of antenna arrays, recently, circular arrays have become the most popular in mobile and wireless communications. In this paper we propose the use of the Taguchi Method to determine an optimum weights and implement it's in an electronic platform using the microcontroller STM8S. 


\section{CIRCULAR ARRAY DESIGN}

The circular array, in which the elements are placed in a circular ring, have an interesting configuration. The array factor of a circular array of $\mathrm{N}$ equally spaced elements is written as [4]:

$$
A F(\theta, \phi)=\sum_{n=1}^{N} I_{n} e^{j\left[k a \sin \theta \cos \left(\phi-\phi_{n}\right)+\alpha_{n}\right]}
$$

Where:

- $N$ : Number of isotropic antenna elements

- $\alpha_{n}=-k a \sin \theta_{0} \cos \left(\phi_{0}-\phi_{n}\right):$ Wave number

- $a$ : Radius of the circular ring

- $I_{n}$ : Amplitude excitation of the $n^{\text {th }}$ element

- $\alpha_{n}$ : Phase excitation of the $n^{\text {th }}$ element

- $\phi_{n}=2 \pi\left(\frac{n}{N}\right)$ : Angular position of the $n^{\text {th }}$ element

To steer the main lobe in the $\left(\theta_{0}, \phi_{0}\right)$ direction, the phase excitation of the $n^{\text {th }}$ element can be chosen to be:

$$
\alpha_{n}=-k a \sin \theta_{0} \cos \left(\phi_{0}-\phi_{n}\right)
$$

The total field of the array is determined by the addition of the fields radiated by the individual elements. Thus, the resulting array factor can be written as:

$$
\begin{aligned}
& A F(\theta, \phi)=\sum_{n=1}^{N} I_{n} e^{j k a\left[\sin \theta \cos \left(\varphi-\varphi_{n}\right)-\sin \theta_{0} \cos \left(\varphi_{0}-\varphi_{n}\right)\right]} \\
& A F(\theta, \phi)=\sum_{n=1}^{N} I_{n} e^{j k a\left[\cos \psi-\cos \psi_{0}\right]}
\end{aligned}
$$

For conformal arrays, the element pattern plays an important role in the array pattern, because each element is facing a different direction [5]. Therefore, the element pattern expression has a different value in each summand of equation 4 .

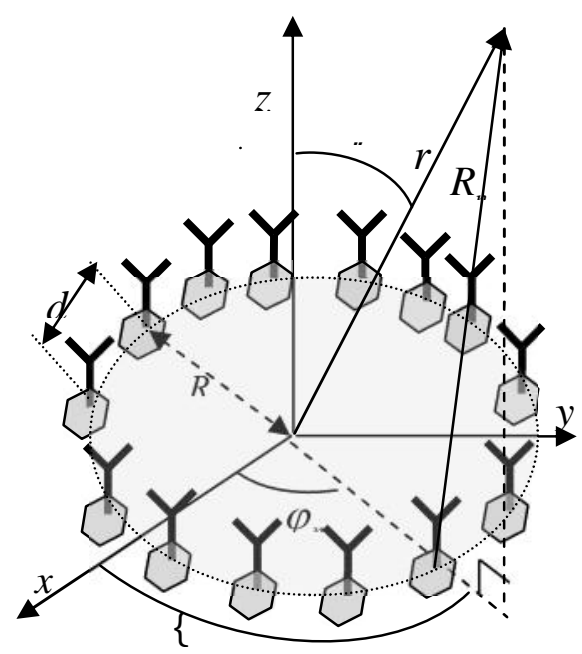

Figure 1. Ceometry of Circular antenna array with $\mathrm{N}$ identical elements 


\section{THE TAGUCHI METHOD}

Taguchi's method was founded on the orthogonal arrays (OA) concept that can actually increase the tests' number needed in an optimization process [6]. The script $O A(N, k, s, t)$ is represented by $\mathrm{N}$ rows, $\mathrm{k}$ columns with s levels and t strengths. The basic benefit of OA concept is that it required only $\mathrm{N}$ experiments to acquire a greater mixture of constant principles. The steps taken in Taguchi's optimization can be summarized as follows [7]-[10]

\subsection{Initialization of the optimization procedure}

In this step, the entry features are chosen for guiding the experiments. For an OA with s equal to 3, the level value 2 for each parameter is selected from the center of the range of optimization for the setting. Then, the other degrees principles are on the same way checked out by removing and adding a "level difference" specific (LD) of the level value of 2 .

The equation which determines the level difference in the first iteration is taken as [7].

$$
L D_{1}=\frac{(\max -\min )}{s+1}
$$

\subsection{The course of experiments}

The fitness value is used to calculate the corresponding signal to noise ratio $(\eta)$ in Taguchi method using this equation :

$$
\eta=-20 \times \log (\text { fitness })
$$

After completion all the experiments and calculate the fitness values and the corresponding signal to noise for each parameter $\mathrm{n}$ and level $\mathrm{m}$ using [11]

$$
\bar{\eta}(m, n)=\frac{s}{N} \sum_{i, O A(i, n)=m} \eta_{i}
$$

With $\mathrm{m}$ is the $m^{\text {th }}$ level and $\mathrm{n}$ is the $n^{\text {th }}$ parameter.

\subsection{Identifying Optimal Level Values and Conducting Confirmation Experiment}

When the response table is calculated, the optimal level for each parameter can be identified by finding the greater signal to noise ration in the corresponding column of the table [12].

After that, a confirmation experiment is made by using the combination of the optimal level values, $\left(V_{n} \mid \begin{array}{l}\text { opt } \\ i\end{array}\right)$ determined in the response table. The fitness value obtained from the optimal combination is considered as the fitness value of the current iteration.

\subsection{Reducing the Optimization Range}

If the termination criteria are not satisfied, the optimal level for the current iteration will be the center of the next iteration.

$$
\left.V_{n}\right|_{i+1} ^{2}=\left.V_{n}\right|_{i} ^{o p t}
$$

In addition, the optimization range for the next iteration is minimized by multiplying the current level difference by the reducing rate $(r r)$ (9). $r r$ can be set between 0,5 and 1 according to the problem [6]. So, for the $(i+1)^{\text {th }}$ iteration.

$$
L D_{i+1}=R R(i) \times L D_{1}=r r^{i} \times L D_{1}
$$

Where $R R(i)=r r^{i}$ is called the reduced function [6] 


\subsection{Checking the Termination Criteria}

The next equation can be used as a termination criterion for optimization procedure [7],[11].

$$
\frac{L D_{i+1}}{L D_{1}} \leq \text { converged value }=0.001
$$

\section{SYNTHESIS AND IMPLEMENTATION}

\subsection{Synthesis radiation patten}

For the purpose of guiding the radiation pattern of a smart antenna in a definite direction, without any power driven or twirl or locomotion, in the synthesis of circular antenna array Taguchi's method will be used by controlling only phase parameters which has 6 equally spaced elements. The elements are spaced by a $\lambda / 2$ and symmetric. The synthesized phases of the 6 elements will be optimized between $-180^{\circ}$ (min) and $180^{\circ}$ (max).

For the 6 elements symmetrical array, with the same amplitude, the array factor can be written as:

$$
A F(\theta, \phi)=2 \sum_{n=1}^{3} e^{j\left(k a\left(\sin (\theta) \cos \left(\varphi-\varphi_{n}\right)-\sin \left(\theta_{0}\right) \cos \left(\varphi_{0}-\varphi_{n}\right)\right)+\varphi_{n}\right)}
$$

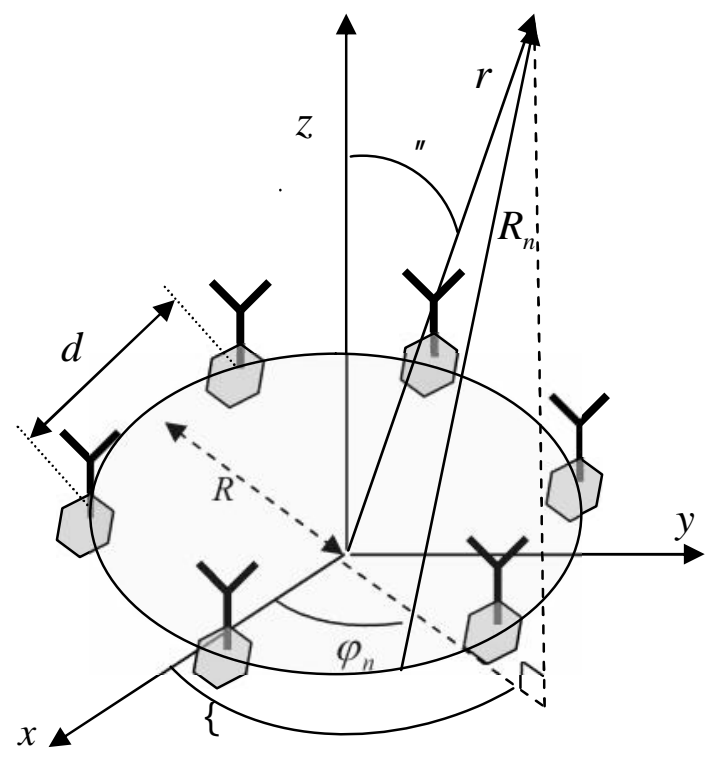

Figure 2. Geometry of 6 elements symmetric circular array

A Laptop with Intel ${ }^{\circledR}$ Pentium ${ }^{\circledR}$ Dual CPU $2 \mathrm{GHz}$ and 2 Go Ram were used for simulating the Taguchi's algorithm, and the simulation time was only 6 seconds. Table 1 shows the simulation results of the proposed approach. Figure 3 present various results of syntheses of circular antenna array at a desired pattern to illustrate the various possibilities offered by the optimization method in order to prove the effectiveness and flexibility of Taguchi method. To illustrate the efficiency of Taguchi's method, the convergence curve of the fitness value is plotted in Figure 4. It is observed that the fitness value converges to the optimum result quickly. 
Table 1. Optimum Phase Values Found by Taguchi's Method

\begin{tabular}{|c|c|c|c|c|}
\hline \multirow{2}{*}{ Element } & \multicolumn{4}{|c|}{ Example : Phase Values } \\
\hline & $(\mathrm{deg}) @-51^{\circ}$ & (deg)@-21 ${ }^{\circ}$ & $(\mathrm{deg}) @ 15^{\circ}$ & $(\mathrm{deg}) @ \mathbf{4 5}^{\circ}$ \\
\hline 1 & $-82,7195$ & $-56,5487$ & $-97,7726$ & $-67,5645$ \\
\hline 2 & 143,4975 & $-107,7140$ & $-45,5329$ & 56,5487 \\
\hline 3 & $-143,4975$ & 107,7140 & 45,5329 & $-56,5487$ \\
\hline 4 & 143,4975 & $-107,7140$ & $-45,5329$ & 56,5487 \\
\hline 5 & $-143,4975$ & 107,7140 & 45,5329 & $-56,5487$ \\
\hline 6 & 82,7195 & 56,5487 & 97,7726 & 67,5645 \\
\hline
\end{tabular}

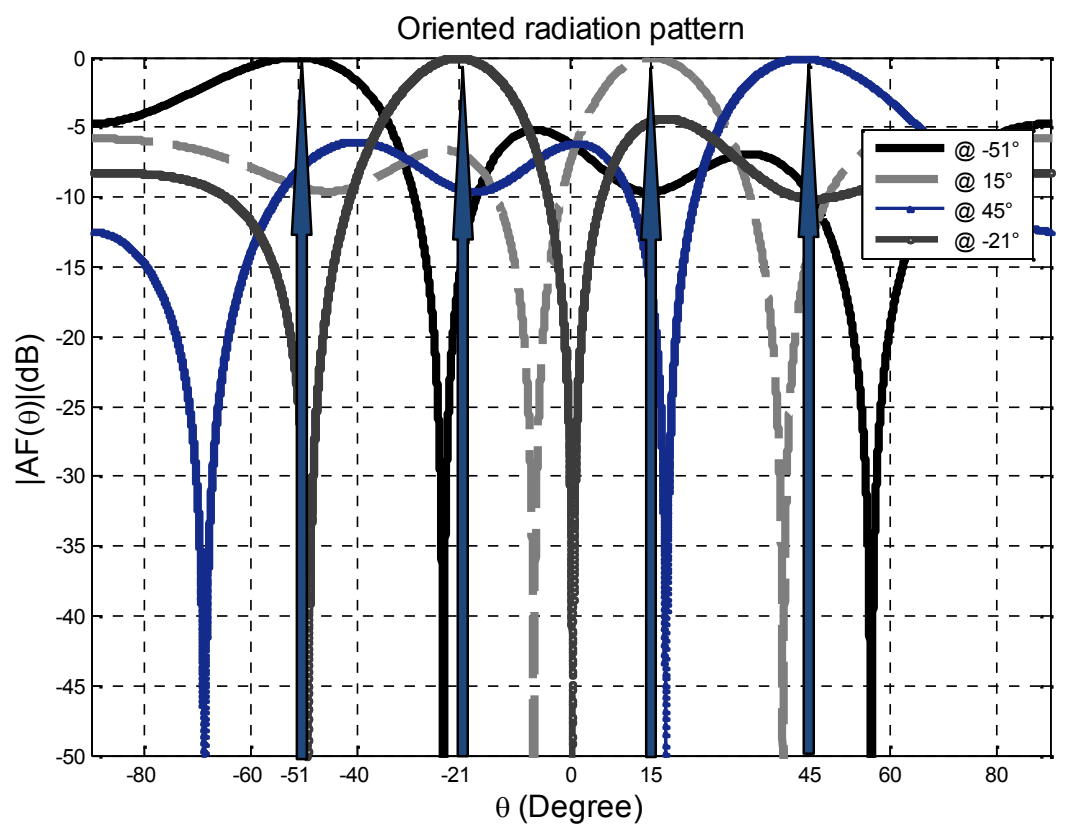

Figure 3. Steering lobes $\left(-51^{\circ}\right),\left(-21^{\circ}\right),\left(15^{\circ}\right)$, and $\left(45^{\circ}\right)$

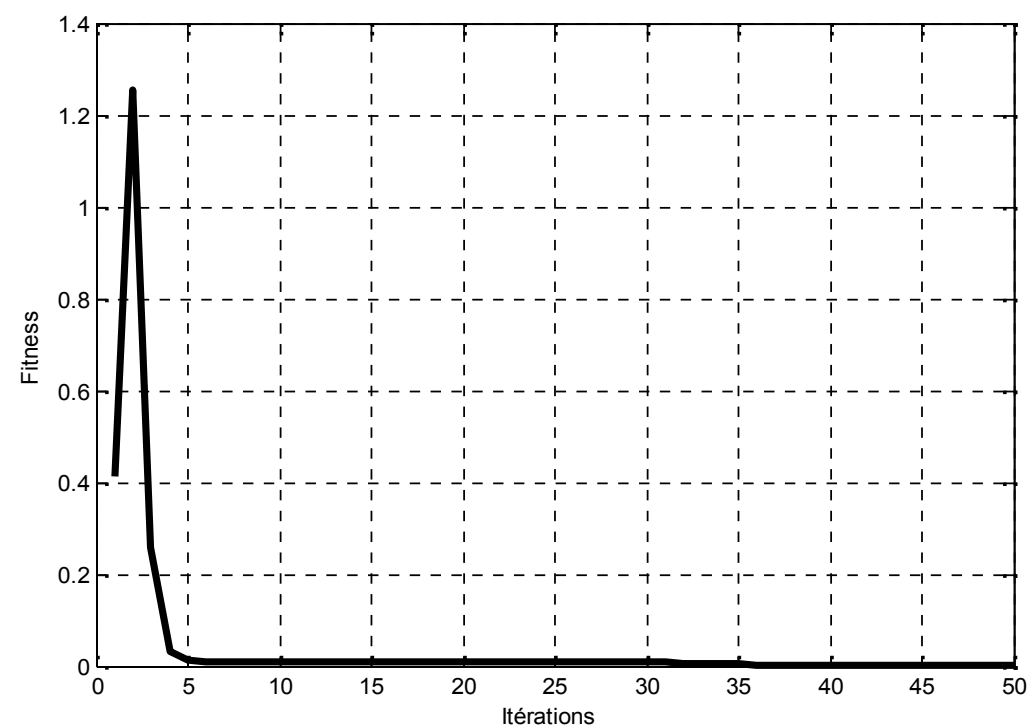

Figure 4. Convergence curve of the fitness value 


\subsection{Implementation of the synthesized phases}

In this section we present an electronic platform dedicated to the implementation of a smart antenna array. Our approach here bring us to a generic architecture, dynamically reconfigurable allowing the direct implementation of the synthesized phases on a STM8S-Discovery which is able to support all the cases of radiation pattern synthesis's [13].

After developing the model of control for the digital phase shifters (8 bits) [14], in the following, we proceed to the implementation for synthesizing phases by the Taguchi algorithm. Our solution is to divide the space into 13 sectors $(\mathrm{S} 1, . ., \mathrm{S} 13)$, repeated every $10^{\circ}$ in the interval from $-65^{\circ}$ to $65^{\circ}$ inclusive (Figure 5), each sector represents a steering lobe. The update of the synthesized weights is given according to the rate of the movement of user which indicates the choice of the sector. Therefore, our implementation has been simulated in the STM8S-discovery built around an STM8S105C6T6 by the C language.

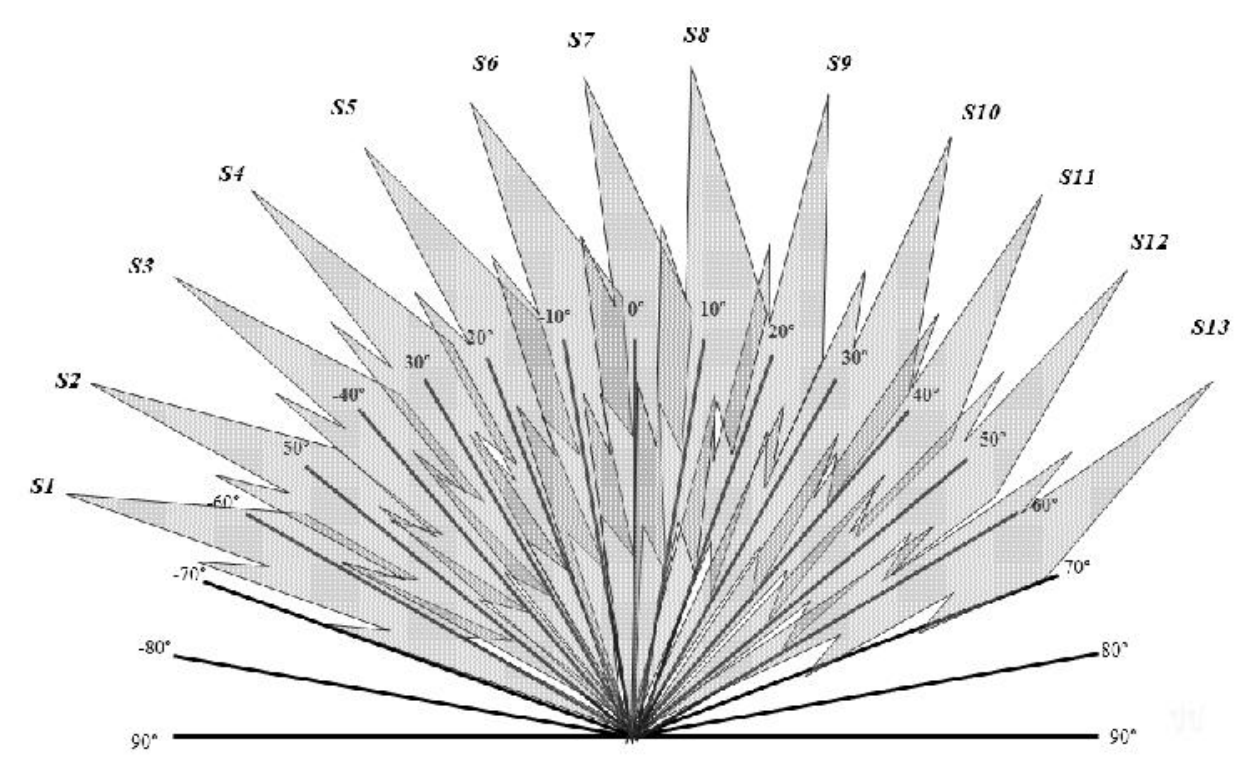

Figure 5. Sector Beamforming

The system consists of 5 parts. A selection input (Sector_lobe) which presents the return of source localization algorithm (MUSIC, ESPRIT...), and indicates the angular position of the user. Once the pointing angle is given, the control device generates the outputs (synthesized phases) which will be the inputs for the digital phase shifters. Then, we find the stage of Modulator/Demodulator, which will be assured by modulators AD8349 in the case of broadcast and by demodulator AD8347 in the case of reception [14].

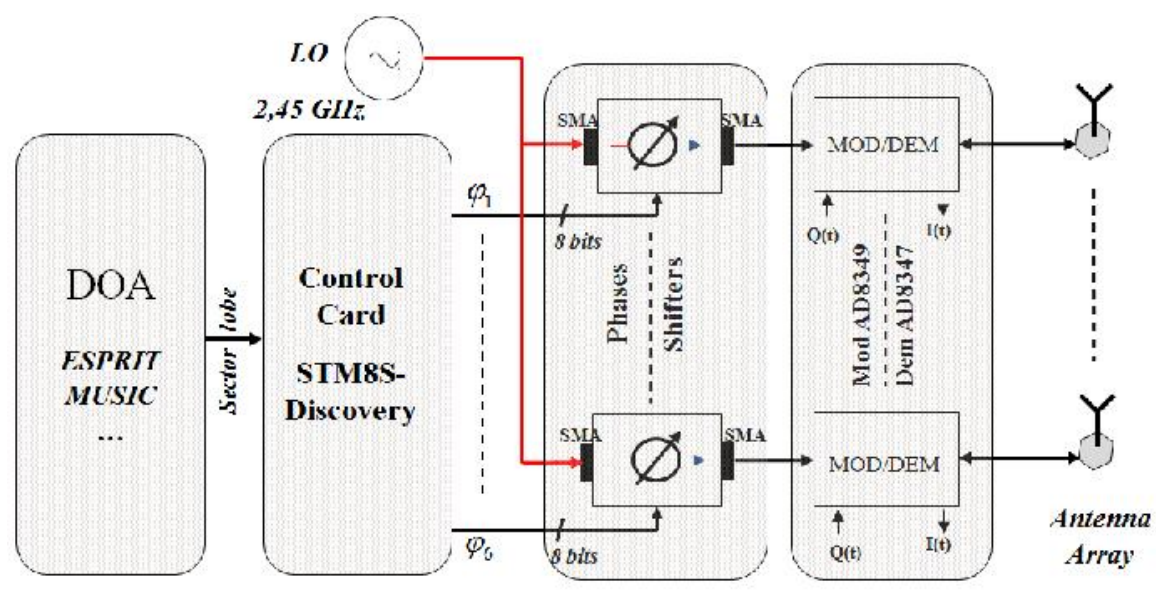

Figure 6. A block diagram of a phased-array antenna composed of antenna elements, phase shifters, and STAM8S Discover card control. 


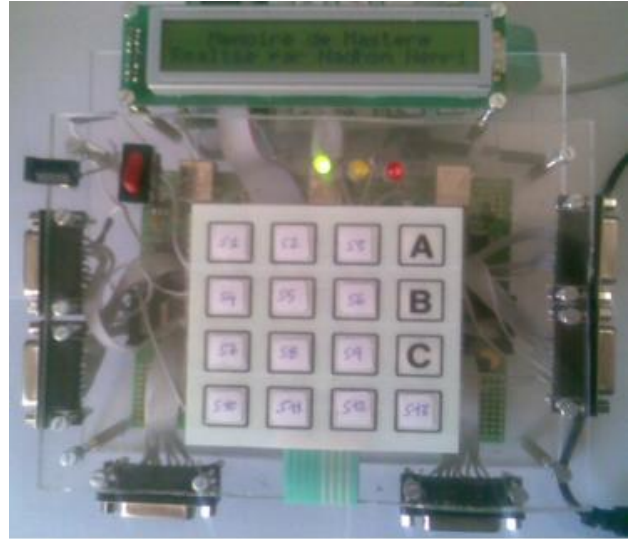

(a)

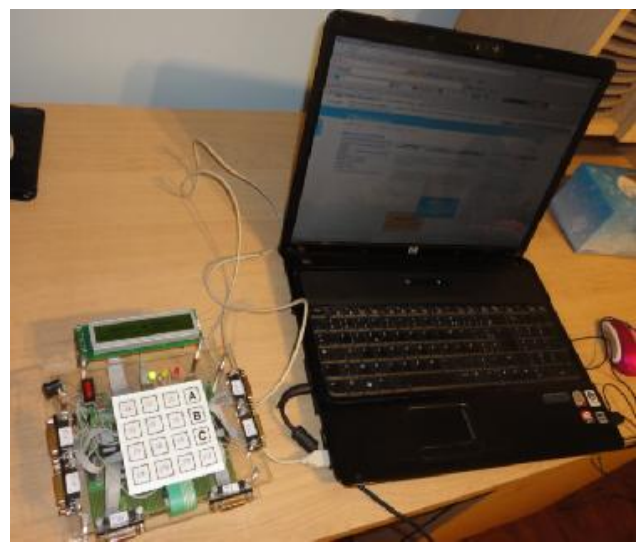

(b)

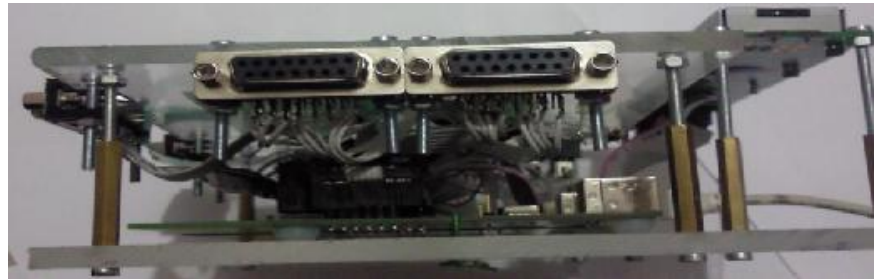

(c)

Figure 7. STM8S-Discovery card control

The RF section includes 6 elementary antennas, to guide the radiation pattern to the desired sector; we must simultaneously excite each antenna by the appropriate phases which are already synthesized with the Taguchi method, so we must use 6 digital phase shifters to realize this. In order to make reliable the total operation of our system, we propose a direct connection between the phase shifters and the ports in/out of the microcontroller. Each phase shifter is controlled with a digital signal ( 8 bits), in total we need 48 bits to satisfy the implementation of all the application. The STM8S105C6 has 48 pins on whom we find 38, which forms the totality of the ports in/out [9] (32 pin configurable as output). With this description we can excite 3 phase shifters with only one microcontroller by using ports B, D and a concatenation of Port A and Port C. So, we need 2 cards STM8S-Discovery to generate 48 bits in the same time.

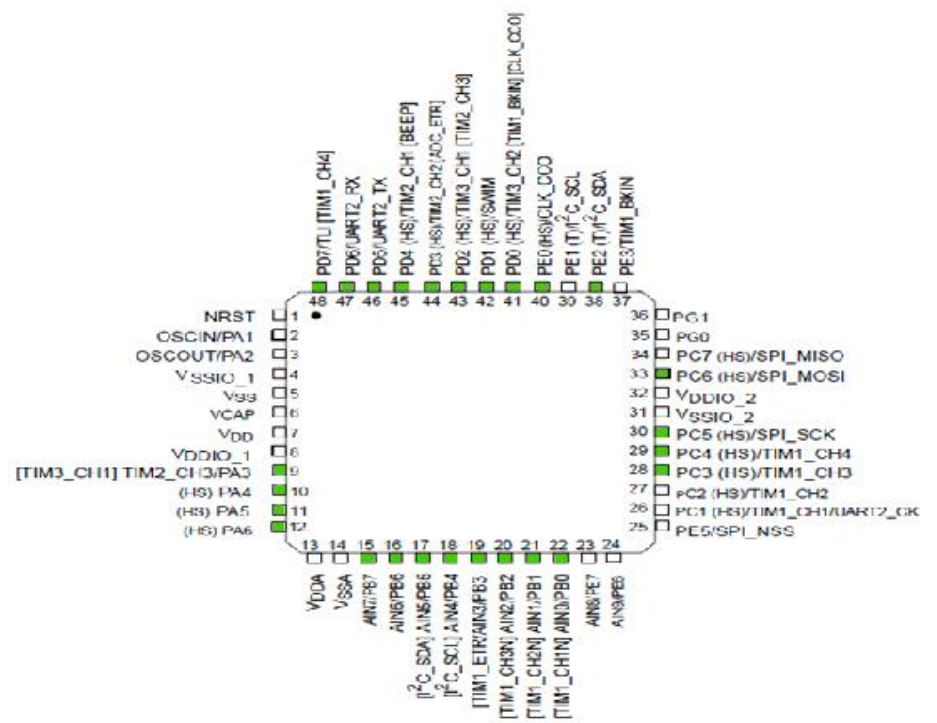

Figure 8. STM8S105C6 : inputs/outputs 
Our solution is to use three cards STM8S-Discovery, two for control generation signals, the third card is for detecting the position of the user who is connected in our case to a keypad which is going to replace the block of estimation arrival directions (DAO). The update of output is done with a test on the input value (sector_lobe) which will be injected in the port B (GPIOB) of the first microcontroller (STM_Master) using a keyboard 16 keys. This reading is managed by a timer interrupt. Periodically the timer creates an interruption, when it occurs; it causes the call of treatment associated. In this routine, the keyboard is readen line by line, its state is coded on 16 bits in a variable that will be decoded and stored in a buffer as a string. Then a test of the buffer's value causes the generation of a synchronization signal ( 8 bits) that is transmitted by a link UART (TX) (serial transmission) with the other cards STM8S. For those cards, the synchronization signal is detected by the UART_RX of each microcontroller; with the value of this signal we get a selection of weights for the update to control the digital phase shifters.

\section{Conclusion}

In this work, a complete analysis and design of a planar circular phased array was proposed. A synthesis method for the digital beamforming using the phase only control with Taguchi method was studied and proposed. This technique based on Taguchi's method was applied on the design of circular antenna arrays and implement Taguchi method in an electronic platform using the microcontroller STM8S. The advantage of Taguchi's optimization technique is the ability of solving problems with a high degree of complexity using small number of experiments in the optimization process. Taguchi's method is easy to implement and converges to the desired goal quickly.

\section{REFERENCES}

[1] R. S. Elliott, “Antenna Theory and design,” Revised Edition, John Wiley, New Jersey, 2003.

[2] N. Pathak, et al., "Synthesis of thinned planar circular array antennas using modified particle swarm optimization," Progress In Electromagnetics Tesearch Lettres, vol. 12, pp. 87-97, 2009.

[3] M. Shihab, et al., "Design of non-uniform Circular antenna arrays using Particle Swarm Optimisation," Journal of ELECTRICAL ENGINERING, vol/issue: 59(4), pp. 216-220, 2008.

[4] C. A. Balanis, "Smart antennas for futur reconfigurable wirless comminication networks," Arizona state University, tech. Rep., 2000.

[5] J. R. Mohamed, "Comparative Performance Investigations of Stochastic and Genetic Algorithms Under Fast Dynamically Changing Environment in Smart Antennas," International Journal of Electrical and Computer Engineering (IJECE), vol/issue: 2(1), pp. 98 105, 2012.

[6] W. C. Weng, et al., "Electromagnetics and Antenna Optimization Using Taguchi's Method," San Rafael, CA: Morgan \& Claypool, 2007.

[7] W. C. Weng, et al., "Linear Antenna Array Synthetis Using Taguchi's Method: A novel optimization in electromaghnetics," IEEE Trans. Antenna Propagation, vol. 55, pp 723-730, 2007.

[8] G. Taguchi, et al., "Taguchi’s Quality Engineering Handbook.,” New York: Wiley, 2005.

[9] R. Roy, "Design of Experiments Using the Taguchi Approach: 16 Steps to product and Process Improvement," John Wiley and Sons, 2001.

[10] S. K. Bodhe, et al., "Beamforming Techniques for Smart Antenna using Rectangular Array Structure," International Journal of Electrical and Computer Engineering (IJECE), vol/issue: 4(2), pp. 257 264, 2014.

[11] W. C. Weng and C. T. M. Choi, "Optimal Design of CPW Slot Antennas Using Taguchi's Method," IEEE Transactions on Magnetics, vol/issue: 45(3), pp. 1542-1545, 2009.

[12] A. Smida, et al., "Amplitude-only adaptative nulling based on Taguchi's method," Multi-Conference on Systems, Signals \& Devices SSD'11, Tunisia, March 22-25, 2011.

[13] N. Nemri, et al., "Phase-Only Array Beam Control using a Taguchi Optimisation Method," Mediterranean Microwave Sympsium (MMS), pp 97-100, September 2011.

[14] N. Nemri, et al., "Implementation of a control system of intelligent antennas based on the Sequential Quadratic Programming (SQP) Algorithm," The 8th European Conference on Antennas and Propagation (EuCAP 2014), pp: 1797-1801, 2014. 


\section{BIOGRAPHIES OF AUTHORS}

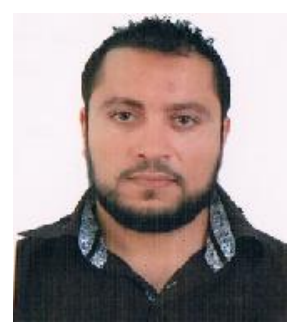

Nadhem NEMRI received the degree in Electronic and science computer Baccalaureate in 2009 and the Master of Science degree in Analyze and Digital Processing of the Electronic Systems in 2011. From 2010 to present, he was a graduate student researcher in the Unit of Research in High Frequency Electronic Circuits and Systems. Since September 2011, he has been a assistant in software engineering and information system department at Higher Institute of Computer Science of El Manar, Tunisia. His current research focuses on smart antennas, Taguchi's method, neural network applications in antennas, adaptive arrays, and difference of arrival, direction of arrival, phased arrays, direction finding.

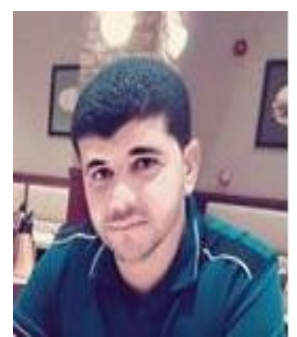

Hani Wadie BADRI received his master's degree in electronics from the Faculty of Sciences, Tunis, Tunisia, in 2011. He is currently working toward his $\mathrm{PhD}$ degree at the Faculty of Sciences, Tunis. His research interests include include wideband antenna

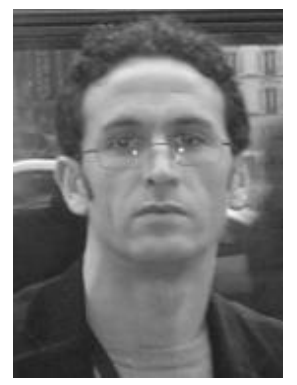

Ridha GHAYOULA received the Diploma Engineer degree in Automatic-Electrical in 2002 and the Master of Science degree in Analyze and Digital Processing of the Electronic Systems in 2005 and the Doctor of Philosophy degree in 2008 from Tunis Manar University,Faculty of Mathematical, Physical and Natural Sciences of Tunis, Tunisia. From 2004 to 2012, he was a graduate student researcher with the Unit of Research in High Frequency Electronic Circuits and Systems. Since August 2009, he has been an assistant professor in the Electrical Engineering Department at Higher Institute of Computer Science of El Manar, Tunisia. Since May 2012, he has been a student researcher in Radiocommunications and Signal Processing Laboratory (LRTS), Department of Electrical and Computer Engineering, Laval University, Québec Canada. His current research focuses on software engineering, field programmable gate array, soft-core processor, modeling and simulation, time difference of arrival, direction of arrival, phased arrays, smart antennas, direction finding, radio communication systems, neural network applications in antennas, adaptive arrays, and microwave circuits design.

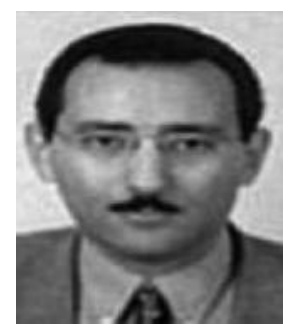

Hichem TRABELSI was born in Tunisia in 1962. He received the PH.D. degree in electronics from the University of Pierre \& Marie Curie, Paris VI, France in 1991. He joined the department of Physics at the Faculty of Sciences, Tunis, in 1992, where he is currently working on microwave active and passive filters and electromagnetic theory for solving field problems in microwave circuits.

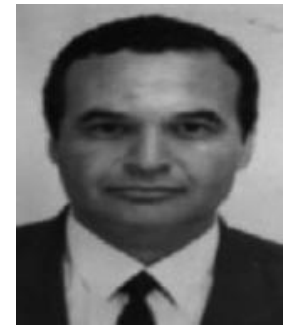

Ali GHARSALLAH received the Radio Frequency Engineering degree from the Higher School of Telecommunication of Tunis, Tunis, Tunisia, in 1986, and the Doctor of Philosophy degree from the Engineering School of Tunis, Tunis, Tunisia, in 1994. Since 1991, he has been with the Faculté des Sciences de Tunis, Department of Physics University Tunis Manar Faculty of Sciences of Tunis, Tunis, Tunisia. He is also a full professor of Electrical Engineering and director of Engineering with the Higher Ministry Education of Tunisia, Tunis, Tunisia. He has authored or coauthored approximately 70 papers published in scientific journals and 120 conference papers. He has also supervised over 20 theses and 50 masters. His current research interests include smart antennas, array signal processing, multilayered structures, and microwave integrated circuits. 